\title{
ECTASIA DA ARTÉRIA BASILAR E ACIDENTE VASCULAR CEREBRAL
}

\author{
ASPECTOS CLÍNICOS DE 21 CASOS
}

\author{
ROBERTO DE MAGALHĀES CARNEIRO DE OLIVEIRA*, \\ JOSÉ OSMAR CARDEAL**, JOSÉ GERALDO DE CAMARGO LIMA***
}

\begin{abstract}
RESUMO - A ectasia da artéria basilar (EB) é a dilatação do calibre em toda ou parte de sua extensão, e/ou quando for anormalmente tortuosa em seu trajeto. As manifestą̧ões clínicas são decorrentes de compressão de nervos cranianos, fenômeno isquêmico ou hemorrágico, síndrome de pseudo-tumor ou hidrocefalia. Procuramos descrever casos da associaçăo de EB e acidente vascular cerebral (AVC), analisar sua frequência, aspectos clínicos e os mecanismos envolvidos nas diferentes formas de apresentação clínica desta entidade. Encontramos 21 pacientes com AVC e EB. A associação de EB e AVC foi prevalente em indivíduos do sexo masculino após 50 anos. Os principais sintomas observados foram hemiparesia, alteração de nervos cranianos e ataxia cerebelar. Na instalação do AVC foram importantes as tonturas rotatórias e a cefaléia. Os infartos relacionados a EB puderam ser atribuídos a diferentes mecanismos: trombose da artéria, embolia artério-arterial, efeito de massa com angulaçāo e obstrução de ramos das artérias vertebrais e basilar.
\end{abstract}

PALAVRAS-CHAVE: acidente vascular cerebral, ectasia, insuficiência vértebro-basilar.

\section{Basilar ectasia and stroke: clinical aspects of 21 cases}

ABSTRACT - Ectasia of the basilar artery (EB) occurs when its diameter is greater than normal along all or part of its course, and/or when it is abnormally tortuous. EB may cause cranial nerve dysfunction, ischemic stroke or subarachnoid hemorrhage, pseudotumor or hydrocephalus. We tried to describe cases of stroke associated with EB, analyze its frequency, clinical aspects, and the mechanisms involved in different forms of its presentation. We found 21 patients with stroke and EB. The association between EB and stroke was more prevalent in males over the age of fifty. Main symptoms were hemiparesia, cranial nerves dysfunction, and cerebellar ataxia. Cerebral infarcts associated with EB were due to different mechanisms: arterial thrombosis, artery-to-artery embolism, mass effect with angulation and obstruction of the vertebral and basilar branches.

KEY WORDS: cerebrovascular disorder, vertebrobasilar insufficiency, ectasia.

Considera-se ectasia da artéria basilar uma dilatação do diâmetro desta artéria em parte ou em toda a sua extensão, e/ou quando for anormalmente tortuosa em seu trajeto ${ }^{30}$. A ectasia da artéria basilar é encontrada na literatura sob vasta sinonímia, que inclui: ectasia da artéria basilar $7,9,17,21,27$, megadolicobasilar ${ }^{15.24}$, malformaçāo megadólico-vascular ${ }^{13}$, dolicoectasia basilar ${ }^{6,16,19,23,25}$, aneurisma cirs6ide (do grego "kirsos" = varizes, "eidos" = aparência) ${ }^{3}$, aneurisma em forma de " $S$ "25, aneurisma fusiforme $e^{411,12,15,18,20,22.28}$, arteria basilar vagante ${ }^{25}$, aneurisma serpentino. $O$ termo megadolicobasilar É mais usado por radiologistas, enquanto patologistas preferem utilizar a expressão ectasia ou aneurisma fusiforme".

O objetivo deste estudo é descrever aspectos clínicos da associação de ectasia da artéria basilar e acidente vascular cerebral (AVC), e analisar os mecanismos envolvidos nas diferentes formas de apresentação clínica desta entidade.

*Disciplina de Neurologia da Universidade Federal de São Paulo (UNIFESP) - Escola Paulista de Medicina: * Pós graduando; ** Professor Adjunto; *** Professor Titular (aposentado). Aceite: 5-maio-1997.

Dr. Roberto de Magalhães Carneiro de Oliveira - Disciplina de Neurologia, Escola Paulista de Medicina - Rua Botucatu 762 - 04023-900 São Paulo SP - Brasil. 


\section{CASUÍSTICA E MÉtOdOS}

Estudamos 21 pacientes com diagnóstico clínico de AVC cuja investigação por imagem revelou ectasia da artéria basilar. Todos os pacientes foram submetidos ao protocolo de investigação para pacientes com AVC no Setor de Doenças Neurovasculares da Disciplina de Neurologia da Escola Paulista de Medicina, no perfodo de 1987 a 1993. O diagnóstico de AVC segue a definição da Organização Mundial da Saúde e a Classificação de Doenças Cerebrovasculares III $^{29}$. Considerou-se $4,5 \mathrm{~mm}$ como limite superior de normalidade de diâmetro da artéria basilar ao nível da ponte, na tomografia computadorizada de crânio (TC) em cortes axiais ${ }^{25}$, e de $3,5 \mathrm{~mm}$, na angiografia cerebral ${ }^{30}$.

\section{RESULTADOS}

Entre 3250 pacientes com AVC encontramos 21 cuja investigação por imagem revelou ectasia da artéria basilar. Em todos o diagnóstico foi feito por TC. Dezenove pacientes $(90,5 \%)$ são do sexo masculino e 2 do sexo feminino (9,5\%). A idade na ocasião do diagnóstico variou entre 36 e 75 anos (média 57,6 anos, mediana 57 anos). Quanto à raça, houve predominância da raça branca, com 14 pacientes $(66,7 \%)$, e apenas 7 da raça negra $(33,3 \%)$.Vinte pacientes $(95,2 \%)$ apresentavam antecedente pessoal de hipertensão arterial, $14(66,7 \%)$ de tabagismo, $14(66,7 \%)$ com uso crônico de bebidas alcoólicas e 4 pacientes $(19,0 \%)$ com diabetes. Oito pacientes $(38,1 \%)$ tinham história de AVC pregresso, 6 de obesidade $(28,6 \%), 3$ de coronariopatia $(14,3 \%), 3$ de doença de Chagas (14,3\%) e 2 de sífilis $(9,5 \%)$.

As manifestações neurológicas encontradas nos pacientes são apresentadas na Tabela 1.

O ecodopplercardiograma e o eletrocardiograma realizados em todos os pacientes não sugeriram fonte cardioembólica em nenhum caso. Todos os pacientes realizaram avaliação por ultrassom das artérias carótidas $e$ apenas 2 pacientes $(9,5 \%)$ apresentaram imagens sugestivas de placas no bulbo carotídeo, sem alteração significante do fluxo.

Quanto ao diagnostico do tipo de AVC, 11 pacientes $(52,4 \%)$ apresentaram AVC lobar (cerebral ou cerebelar) e igualmente 11 pacientes apresentaram AVC lacunar. O diagnóstico vascular cerebral mais comum encontrado nos pacientes com ectasia da artéria basilar foi o AVC isquêmico $(95,2 \%)$, seguido pelos ataques isquêmicos transitórios $(14,3 \%)$, hemorragia subaracnoídea $(4,8 \%)$ e AVC hemorrágico $(4,8 \%)$. Nos 2 pacientes que apresentaram hemorragia subaracnoídea também evidenciou-se a ocorrência de infarto (AVCI).

\section{DISCUSSÃO}

Tendo encontrado 21 pacientes com ectasia da artéria basilar entre 3250 pacientes com AVC, podemos considerar esta uma associação rara. Estudos retrospectivos de necrópsias ${ }^{18}$ descrevem a ectasia da artéria basilar como raridade. Com o advento de exames de imagem não invasivos, observouse a identificação mais frequente desta entidade, passando a diagnosticar-se também os assintomáticos. Há carência de estudos epidemiológicos que permitam a determinação da incidência de ectasia da artéria basilar em amostra populacional significante, que incluissem indivíduos assintomáticos.

Observamos a associação entre ectasia da artéria basilar e AVC quase exclusivamente em homens acima dos 50 anos de idade, o que é concordante com outros autores ${ }^{17,18.30}$. Não há, até o momento, explicação para a maior frequência em indivíduos do sexo masculino. Não observamos nenhum caso em criança. Os raros relatos de ectasia da artéria basilar em crianças e jovens têm sido associados à origem congênita (doença de Anderson-Fabry, síndrome de Marfan, doença de Pompe, pseudoxantoma elástico, síndrome de Menkes e deficiência de $\alpha$-glicosidase) $)^{11,12,14,15,22,24}$.

Encontramos elevada proporção de indivíduos hipertensos $(95,2 \%)$, acima da maioria das séries publicadas ${ }^{10,19}$. A hipertensão arterial parece ter papel importante, mas não exclusivo, na gênese da ectasia arterial. A aterosclerose não foi importante em nossos pacientes. Antecedente de sífilis pregressa em dois pacientes pode estar relacionada ao desenvolvimento da ectasia arterial ${ }^{12,27}$. 
Tabela 1. Distribuição das manifestações neurológicas observadas durante a instalação do AVC nos pacientes com ectasia da artéria basilar.

\begin{tabular}{|c|c|c|}
\hline Manifestaçāo neurológica & $\mathbf{n}$ & $\%$ \\
\hline Deficit motor (hemiparesia) & 13 & 61,9 \\
\hline Alteraçăo de nervos cranianos & 11 & 52,4 \\
\hline N. vago & 6 & 28,6 \\
\hline N. vestibulo-coclear & 5 & 23,8 \\
\hline N. facial & 5 & 23,8 \\
\hline N. trigêmeo & 4 & 19,0 \\
\hline N. glossofaringeo & 4 & 19,0 \\
\hline N. abducente & 2 & 9,5 \\
\hline N. acessório & 1 & 4,8 \\
\hline N. hipoglosso & 1 & 4,8 \\
\hline Tonturas rotatórias & 11 & 52,4 \\
\hline Comprometimento cerebelar & 8 & 38,1 \\
\hline Ataxia & 8 & 38,1 \\
\hline Dismetria & 6 & 28,6 \\
\hline Fala escandida & 2 & 9,5 \\
\hline Cefaléia & 7 & 33,3 \\
\hline Hipoestesia & 5 & 23,8 \\
\hline Disartria & 4 & 19,0 \\
\hline Hipomnésia & 4 & 19,0 \\
\hline Riso imotivado & 2 & 9,5 \\
\hline Apatia & 2 & 9,5 \\
\hline Afasia de expressão & 1 & 4,8 \\
\hline Apraxia ideo-motora & 1 & 4,8 \\
\hline Quadrantopsia homônima & 1 & 4,8 \\
\hline Paresia do olhar conjugado para cima & 1 & 4,8 \\
\hline Acalculia & 1 & 4,8 \\
\hline Agnosia visual & 1 & 4,8 \\
\hline "Drop attacks" & 1 & 4,8 \\
\hline
\end{tabular}

Pudemos observar duas formas de apresentação dos sintomas em nossos pacientes, alguns com espasmo de hemiface há anos, desenvolvendo AVC mais recentemente, e outros inaugurando com o quadro vascular. Ao contrário do que alguns autores relatam ${ }^{2.26}$, em nossa casuística o nervo craniano mais acometido foi o nervo vago, possivelmente pelo critério de seleção dos pacientes, a associação de AVC e ectasia da artéria basilar. Disfonia, disfagia e assimetria no reflexo faringeo foram as manifestaçōes mais observadas nestes pacientes. O segundo nervo mais acometido foi o nervo vestrbulo-coclear. Nestes pacientes pode-se encontrar hipoacusia, zumbidos pulsáteis ou vertigem como na síndrome de Ménière ${ }^{2}$. O ponto de acometimento do nervo ocorre na sua emergência no tronco cerebral, onde há a transição entre a bainha de mielina do tipo periférica para a mielina do tipo central, na chamada zona de Oberstein-Redlich.

Dois pacientes apresentavam antecedentes de espasmo hemifacial de provável etiologia compressiva, um deles desenvolvendo posteriormente paralisia facial periférica. Quatro pacientes apresentaram paralisia facial periférica, não sendo possivel, em alguns deles, a determinaçāo exata do mecanismo envolvido (compressivo ou isquêmico). Smoker e col. estudaram 22 pacientes com espasmo hemifacial por TC, e encontraram ectasia da artéria basilar em todos os pacientes ${ }^{26}$. Para muitos autores o nervo facial é o mais acometido pela ectasia da artéria basilar, ${ }^{6,1926}$. Encontramos 4 pacientes com hipoestesia facial e nenhum caso de nevralgia do trigêmeo. $O$ nervo trigêmeo é tão frequentemente acometido pela ectasia da artéria basilar quanto o nervo facial. 
Dois pacientes apresentavam défícits do nervo abducente associados a outros sinais de comprometimento do tronco cerebral. A artéria basilar dilatada ou alongada e tortuosa pode comprimir o nervo abducente no espaço subaracnóide, ou provocar a tração neste nervo secundariamente a uma rotação do tronco cerebral ${ }^{25}$.

Dentre as diferentes formas de AVC, encontramos o AVC isquêmico em 95,2\% de nossos pacientes, sendo o principal tipo de AVC em pacientes com ectasia de artéria basilar. A frequência deste tipo de manifestação varia com os autores, entre $30 \%$ e $40 \%$ 2.19,26. Quatro pacientes $(19,0 \%)$ apresentavam sinais de trombose parcial da artéria basilar à investigação por métodos de imagem. $A$ dilatação do sistema vascular é responsável pela estase, agravada pela tortuosidade, que causando lentificação do fluxo sanguíneo, propiciam a trombose. Seis $(28,6 \%)$ pacientes apresentaram infartos em territorio distal de circulaçāo posterior, sugerindo mecanismo embólico artério-arterial. Para que haja embolia artério-arterial, deve ocorrer combinação de fluxo lentificado e turbilhonante, lesões ateromatosas e migração do trombo. Alguns autores sugerem o mecanismo embólico a partir de uma artéria basilar dilatada para o infarto em território da artéria cerebral posterior. Três pacientes apresentaram infarto talâmico distal à artéria basilar dilatada, que tem sido descrito como consequente à embolia pelas artérias perfurantes talâmicas a partir do topo da artéria basilar ${ }^{4}$. Onze pacientes $(52,4 \%)$ apresentaram AVC em território vértebro-basilar, onde o mecanismo pode ter sido a angulação de ramos das artérias vertebrais e basilar. Este mecanismo envolvido na ocorrência do infarto no tronco cerebral, em pacientes com ectasia da artéria basilar, é decorrente da distorção e obstruçāo de ramos paramedianos da artéria basilar, acrescentando-se a isto, a rotação do tronco cerebral provocada pela compressão da artéria dilatada, e a angulação dos ramos da artéria basilar tortuosa e deslocada de seu eixo normal ${ }^{19}$. A isquemia pode ser secundária ao efeito de massa direto produzido pela grande ectasia ${ }^{20}$. Podemos notar que mais de um mecanismo pode estar envolvido no desenvolvimento de infartos no cérebro de pacientes com ectasia da artéria basilar. Onze pacientes $(52,4 \%)$ apresentaram imagens sugestivas de infartos lacunares, provavelmente secundários à lipo-hialinose arteriolar, consequência da hipertensāo arterial.

Encontramos $52,4 \%$ dos pacientes com tontura rotatória precedendo ou concomitante ao AVC, geralmente acompanhada por vômitos, que foi atribuida à insuficiência vértebro-basilar.

Os sintomas mais frequentes em nossa casuística foram a hemiparesia (61,9\%), alteração de nervos cranianos $(52,4 \%)$ e comprometimento de cerebelo ou de vias cerebelares $(38,1 \%)$. A associação de sinais piramidais e cerebelares encontrada em 4 pacientes $(19,0 \%)$ e comum em pacientes com ectasia da artéria basilar ${ }^{17}$. A perda visual é incomum, e decorre da compressão optoquiasmática, do trato 6́ptico, ou compressão do lobo occipital, ou ainda por infarto embólico a partir da artéria basilar dilatada. Observamos 3 casos de hemiparesia ipsilateral aos déficits de nervos cranianos e cerebelo, que podem ser explicados pela compressão direta ou indireta de nervos cranianos e do tronco cerebral pela artéria ectasiada.

Três pacientes $(14,3 \%)$ apresentaram episódios de isquemia transitória. Os ataques isquêmicos transitórios têm sido frequentemente descritos em pacientes com ectasia da artéria basilar ${ }^{19,20}$. A forma de apresentação destes episódios transitórios inclui pelo menos dois destes achados: cefaléia, tontura, fala escandida, diplopia, astasia, ataxia e hemiparesia ${ }^{20}$.

Encontramos 2 pacientes com história clínica e exame de líquido cefalorraqueano compatíveis com hemorragia subaracnoídea, e com angiografia cerebral sem aneurisma sacular. A hemorragia subaracnóidea resultante da ruptura de artéria vertebral ou basilar ectasiada tem sido considerada rara $^{1.21}$, embora relatos tenham documentado esta complicação ${ }^{12.20}$. Não é ilógico esperar que uma artéria com parede dilatada e afilada possa ceder e romper-se, pois um defeito estrutural do vaso, que não aterosclerose, pode predispor a esta complicação. Estudos post mortem têm descrito pacientes com hemorragia subaracnoídea e defeito estrutural no vaso como deficiência de elastina, displasia fibrosa da íntima e degeneração da lâmina elástica interna ${ }^{13}$, deficiência de $\alpha$-glicosidase ${ }^{15}$.

Como escolhemos a presença de AVC como critério de seleção dos pacientes, nenhum paciente apresentou apenas o quadro clínico de pseudo-tumor. A artéria basilar dilatada pode apresentar-se como processo tumoral, principalmente no ângulo ponto-cerebelar, podendo mesmo simular um tumor desta regiãos. Pode também mimetizar lesāo do tronco cerebral ou de um hemisfério cerebelar ${ }^{2}$ 
através da compressāo destas estruturas, promovendo a desmielinização dos tratos nervosos ${ }^{22}$, ou interferindo com sua irrigaçẳo normal ${ }^{23}$. Muitas vezes a artéria se apresenta tortuosa em forma de "S", provocando compressão nos dois lados do tronco cerebral. A artéria basilar dilatada pode causar a atrofia do bulbo e do trato piramidal ou a erosão da ponte ${ }^{4,11}$.

Um terço de nossos pacientes apresentou cefaléia intensa à instalação do AVC. Frequentemente é mencionada cefaléia em pacientes com ectasia da artéria basilar. Dependendo dos autores sua causa varia de trombose e isquemia ${ }^{19}$ a compressāo ou estiramento da vasa vasorum ${ }^{2}$. A cefaléia é comumente posterior ou nucal, pulsátil ou constritiva.

Diante da diversidade de expressão clínica da ectasia da artéria basilar, seu diagnóstico não pode ser feito com base clínica exclusivamente, necessitando de métodos de diagnóstico por imagem ${ }^{1,17}$.

\section{REFERÊNCIAS}

1. Boeri R, Passerini A. The megadolichobasilar anomaly. J Neurol Sci 1964;1:475-484.

2. Brichaux JC, Gense D, Greselle JF, Jasek F, Bouin H, Caillé JM. Problèmes radiocliniques posés par les méga-dolicho trones basilaires: à propos de 17 observations et revue de la littérature. J Neuroradiol 1989;16:11-24.

3. Campbell E, Keedy C. Hemifacial spasm: a note on the etiology in two cases. J Neurosurg 1947;4:342-347.

4. Echiverti HC, Rubino FA, Gupta SR, Gujrati M. Fusiform aneurysm of the vertebrobasilar arterial system. Stroke 1989;20:1741-1747.

5. Frasson F, Ferrari G, Fiaschi A. Megadolichobasilar anomaly causing brainstem syndrome.Neuroradiology 1977;13:279-281.

6. Gautier JC, Hauw JJ, Awada A, Loron P, Gray F, Juillard JB. Artères cérébrales dolichoectasiques: association aux anévrysmes de l'aorte abdominale. Rev Neurol (Paris) 1988;144:437-446.

7. Hassler $\mathrm{O}$. Morphological studies on the large cerebral arteries with reference to the aetiology of subarachnoid haemorrhage. Acta Psychiatr Neurol Scand 1961;36(Suppl 154):5-145.

8. Hatano S. Experience from a multicenter stroke register: a preliminary report. Bull World Health Organ 1976;54:541-553.

9. Hegedus K. Ectasia of the basilar artery with special reference to possible pathogenesis. Surg Neurol 1985;24:463-469.

10. Herpers M, Lodder J, Janevski B, Van Der Lugt PJM. The symptomatology of the megadolicho basilar artery. Clin Neurol Neurosurg 1983;85:203-212.

11. Hirsch CS, Roessmann U. Arterial dysplasia with nuptured basilar artery aneurysm: report of a case. Hum Pathol 1975;6:749-758.

12. Johnsen SD, Okamoto G, Kooiker J. Fusiform basilar artery aneurysm in a child. Neurology 1977;27:334-336.

13. Lodder J, Janevski B, Van Der Lugt PJM. Megadolicho vascular malformation of the intracranial arteries. Clin Neurol Neurosurg 1981;83:11-18.

14. Maisey DN, Cosh JA. Basilar artery aneurysm and Anderson-Fabry disease. J Neurol Neurosurg Psychiatry 1980;43:85-87.

15. Makos MM, McComb RD. Hart MN, Bennett DR. Alpha-glucosidase deficiency and basilar artery aneurysm: report of a sibship. Ann Neurol 1987;22:629-633.

16. Milandre L, Bonnefoi B, Pestre P, Pellissier JF, Grisoli F, Khalil R. Dolichoectasies artérielles vertébrobasilaires: complications et prognostic. Rev Neurol (Paris) 1991;147:714-722.

17. Moseley I F, Holland IM. Ectasia of the basilar artery: the breadth of the clinical spectrum and the diagnostic value of computed tomography. Neuroradiology 1979;18:83-91.

18. Nijensohn DE, Saez RJ, Reagan TJ. Clinical significance of basilar artery aneurysms. Neurology 1974;24:301-305.

19. Nishizaki T, Tamaki N, Takeda N, Shirakuni T, Kondoh T, Matsumoto S. Dolichoectatic basilar artery: a review of 23 cases. Stroke 1986;17:1277-1281.

20. Pessin M S, Chimowitz MI, Levine SR, Kwan E S, Adelman LS, Earnest MP, Clark DM, Chason J, Ausman JI, Caplan LR. Stroke in patients with fusiform vertebrobasilar aneurysms. Neurology 1989;39:16-21.

21. Peterson NT, Duchesneau PM, Westbrook EL, Weinstein MA. Basilar artery ectasia demonstrated by computed tomography. Radiology 1977;122:713-715.

22. Read D, Esiri MM. Fusiform basilar artery aneurysm in a child. Neurology 1979;29:1045-1049.

23. Sacks JG, Lindenberg R. Symptomatology and pathology of arterial elongation and distention. Hopkins Med J 1969:125:95-106.

24. Scotti G, De Grandi C, Colombo A. Ectasia of the intracranial arteries diagnosed by computed tomography: megadolichobasilar artery CT diagnosis. Neuroradiology 1978;15:183-184.

25. Smoker WR, Price MJ, Keyes WD, Corbett JJ, Gentry LR. High-resolution computed tomography of the basilar artery:1. Normal size and position. AJNR 1986;7:55-60.

26. Smoker WR, Corbett JJ, Gentry LR, Keyes WD, Price MJ, McKusker S. High-resolution computed tomography of the basilar artery: 2. Vertebrobasilar dolichoectasia: clinical-pathologic correlation and review. AJNR 1986;7:61-72.

27. Wallace S, Jaffe ME. Cerebral arterial ectasia with saccular aneurysms. Radiology 1967;88-90-93.

28. Watanabe T. Sato K, Yoshimoto T. Basilar artery occlusion caused by thrombosis of atherosclerotic fusiform aneurysm of the basilar artery. Stroke 1994;25:1068-1070.

29. Whisnant JP, Basford JR, Bernstein EF, et al.. Classification of cerebrovascular disease III. Stroke 1990;21:637-676.

30. Yu Y, Moseley I, Pullicino P, McDonald W. The clinical picture of ectasia of the intracerebral arteries. J Neurol Neurosurg Psychiatry 1982;45:29-36. 